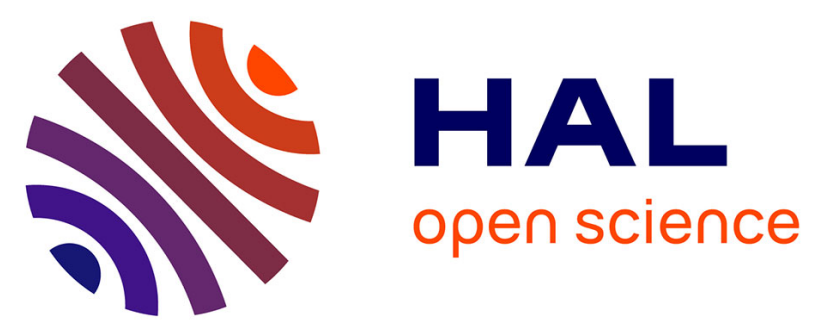

\title{
Time and spatial resolved optical and electrical characteristics of continuous and time modulated RF plasmas in contact with conductive and dielectric substrates
}

Sven Hofmann, Koen Van Gils, Steven van Der Linden, Sylvain Iseni, Peter

Bruggeman

\section{To cite this version:}

Sven Hofmann, Koen Van Gils, Steven van Der Linden, Sylvain Iseni, Peter Bruggeman. Time and spatial resolved optical and electrical characteristics of continuous and time modulated RF plasmas in contact with conductive and dielectric substrates. The European Physical Journal D: Atomic, molecular, optical and plasma physics, 2014, 68 (3), pp.56-56. 10.1140/epjd/e2014-40430-3 . hal02270234

\section{HAL Id: hal-02270234 \\ https://hal.science/hal-02270234}

Submitted on 26 Jun 2021

HAL is a multi-disciplinary open access archive for the deposit and dissemination of scientific research documents, whether they are published or not. The documents may come from teaching and research institutions in France or abroad, or from public or private research centers.
L'archive ouverte pluridisciplinaire HAL, est destinée au dépôt et à la diffusion de documents scientifiques de niveau recherche, publiés ou non, émanant des établissements d'enseignement et de recherche français ou étrangers, des laboratoires publics ou privés. 


\title{
Time and spatial resolved optical and electrical characteristics of continuous and time modulated RF plasmas in contact with conductive and dielectric substrates
}

\author{
Sven Hofmann, Koen van Gils, Steven van der Linden, Sylvain Iseni ${ }^{\mathrm{a}}$, and Peter Bruggeman ${ }^{\mathrm{b}}$ \\ Department of Applied Physics, Elementary Processes in Gas Discharges, Eindhoven University of Technology, \\ P.O. Box 513, 5600 MB, Eindhoven, The Netherlands
}

Received 19 July 2013 / Received in final form 15 November 2013

Published online 21 March 2014

\begin{abstract}
Cold atmospheric pressure plasma jets are often used as a remote plasma source for substrate treatments. However, this substrate acts as an electrode and this additional electrode can induce effects on plasma parameters such as the dissipated power, gas temperature, etc. In this work the influence of substrates of different conductivity and permittivity in direct contact with three different operational modes of atmospheric pressure RF plasma jets is investigated. Two different electrode configurations (creating either a linear or a cross electric field) and, for the linear field configuration, two voltage modulations (continuous RF and $\mathrm{kHz}$ pulsed $\mathrm{RF}$ ) have been studied. Electrical and optical diagnostic methods have been performed in order to get quantitative data of the change in plasma dissipated power and gas temperature, when the plasma is in direct contact with the substrate. In all three investigated cases the power dissipation and gas temperature, significantly increase when the plasma is in direct contact with a conductive substrate. The increase of power is attributed to a change of the equivalent electrical circuit, leading to a more favourable matching between the power input and the plasma source.
\end{abstract}

\section{Introduction}

Cold atmospheric pressure plasmas (CAPPs) are nonequilibrium plasmas with a gas temperature as low as room temperature, while still maintaining an electron temperature of a few electron volt. This feature allows them to produce reactive chemistry at low gas temperatures and makes them applicable for treatment of heat sensitive materials [1].

In the past years cold atmospheric pressure plasmas have been shown to be a promising tool for applications in medicine such as inactivation of bacteria (e.g. Methicillin resistant $S$. aureus) or direct treatment of living tissue [2-11]. Different types of configurations can be used to create CAPPs, ranging from DC, kHz-pulsed dielectric barrier discharges (DBDs) to nanosecond pulsed, radio frequency or microwave driven plasma jets [12-14].

Depending on the type of discharge and the application, the plasma is either in direct contact with the treated substrate or not. Except for surface DBDs and coplanar DBDs (see e.g. $[15,16]$ ), for most applications based on dielectric barrier discharges the treated substrate

\footnotetext{
${ }^{a}$ Current address: Leibniz Institute for Plasma Science and Technology INP Greifswald e.V., Felix-Hausdorff-Str. 2, 17489 Greifswald, Germany

b e-mail: pbruggem@umn.edu
}

acts as one of the electrodes and is needed to create the plasma $[15,17,18]$. The disadvantage of those sources is that the plasma itself depends strongly on the characteristics of the substrate, such as conductivity, permittivity and smoothness of the treated area, which makes diagnostics of plasma parameters challenging [19].

In a plasma jet, however, the plasma is in most cases remotely created between two electrodes in a noble gas $[12,13]$. Due to the electric field and the gas flow, the plasma created species are then forced out of the plasma zone in the tube into the open air. The advantage with these devices is that the treated substrate is not needed in order to sustain the discharge, which means that the plasma can either directly (in contact) or indirectly treat the material.

It is commonly believed that the main difference between a direct and indirect treatment is that in the latter case, only long living species are able to reach the substrate, while in the first case also charged species, (V)UV and the electric field will interact with the substrate [20]. However, as in the case of a dielectric barrier discharge, the moment the plasma is close to or in contact with a substrate, the substrate itself will act as an additional electrode, changing the electric circuit and thereby altering the plasma properties itself. How much the plasma changes depends on the conductivity of the substrate and the configuration of the plasma source [7,21-23]. 
Table 1. Typical electrical conductivity $(\sigma)$ and relative permittivity $\left(\epsilon_{r}\right)$ values of the substrates used in this work. [*] measured.

\begin{tabular}{ccc}
\hline Substrate & $\sigma[\mathrm{S} / \mathrm{m}]$ & $\epsilon_{r}$ \\
\hline Tap water & $\left.0.025{ }^{*}\right]$ & 80, distilled at $1.6 \mathrm{GHz}$ \\
& & and $20^{\circ} \mathrm{C}[28]$ \\
Aluminium & $10^{7}[29]$ & - \\
Glass (BK7) & $10^{-13}[30]$ & 4.9 at $1 \mathrm{MHz}[31]$ \\
\hline
\end{tabular}

In most cases the influence of the substrate on the plasma itself has been investigated using imaging. Results indicate that a plasma in contact with an insulator shows no significant change of the plasma while touching a conductive substrate does increase the intensity of the emission and in some cases the length of the plasma jet plume [24].

Simulations of a helium plasma needle show that particle fluxes depend strongly on the treated substrate. The reason for that is the altered configuration of the electric field which will change the flux of charged particles onto the substrate [22]. Experimentally these simulations have been compared with images of the visible plasma jet and power measurements as a function of the distance between the plasma and the treated substrate, in this case agar (a gel used for bacteria and fungi growth) $[21,25]$.

One example of a diagnostic method which can influence the plasma is molecular beam mass spectrometry (MBMS). MBMS usually samples the plasma with a metal orifice and this will change the plasma properties completely when the plasma is in direct contact with the metal orifice, compared to the free plasma. The results obtained from these experiments have to be interpreted with care as observed and mentioned, e.g. in references [26,27].

As in most in vitro biological treatments water and saline-like solutions are present, understanding how the plasma behaves in contact with liquids is very important.

In this work we apply in situ measurements of the plasma dissipated power and the gas temperature, when the plasma is in contact with a substrate, i.e. water, glass and metal. The typical values of permittivity and conductivity of the interfaces used are shown in Table 1 . The observed parameters have been obtained by time resolved ICCD imaging and time resolved plasma dissipated power measurements combined with spatially resolved optical emission spectroscopy. An RF argon plasma jet with two different electrode configurations and time modulated and continuous RF excitation has been used.

The paper is structured as followed. In Section 2 the plasma source and the diagnostics are introduced. Section 3 deals with the results starting with obtained CCD images, followed by electrical characteristics and ICCD images (Sect. 4) and gas temperature measurements of the plasma in contact with water (Sect. 5). In addition, these results are compared with measurements performed with a glass and an aluminium substrate (Sect. 6). In Section 7 a summary of the results is given and we discuss the consequences of the presented research for related studies.

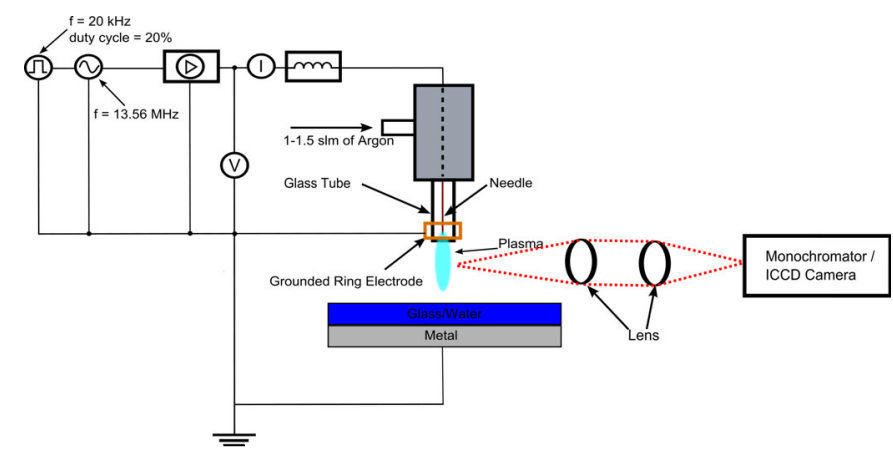

Fig. 1. Experimental setup.

\section{Setup and methods}

Figure 1 shows a schematic view of the setup. An RF voltage with a frequency of $13.56 \mathrm{MHz}$ generated with a waveform generator (Agilent 33220A) and amplified by a power amplifier (Amplifier Research 75AP250) is applied to the needle electrode. The output of the amplifier is connected to an inductance, which is close to resonance with the jet at a frequency of $13.56 \mathrm{MHz}$. This simple network design has been chosen in order to reduce the amount of impedances in the circuit connected to ground. These need to be otherwise considered when correcting for the power which is dissipating in the matching network (see power measurements below and [32]). The matching network is connected to the plasma source, which consists of a needle electrode $(\varnothing=1 \mathrm{~mm})$ in the centre of a dielectric enclosing. The needle is surrounded by a quartz glass tube, with an inner diameter of $1.7 \mathrm{~mm}$ and an outer diameter of $3 \mathrm{~mm}$. An argon flow of $1 \mathrm{slm}$ through the glass tube is controlled by a mass flow controller (Brooks $5850 \mathrm{E}$ calibrated for $0-3 \mathrm{slm}$ of helium) connected to a control unit (Brooks 0254).

For the grounded electrode either a ring electrode surrounding the glass tube or a plate electrode in front of the glass tube is used. These configurations result in either an electric field perpendicular (cross-field configuration) or parallel to the gas flow (linear-field configuration) [33]. The ring electrode has a length of $5 \mathrm{~mm}$, the end of the ring electrode is $2 \mathrm{~mm}$ behind the needle electrode and $0.5 \mathrm{~mm}$ before the end of the glass tube. The plate electrode is at a distance of $4 \mathrm{~mm}$ away from the needle electrode and $2 \mathrm{~mm}$ away from the end of the glass tube. The plate has a hole with a diameter of $5 \mathrm{~mm}$ to let the gas flow and the plasma penetrate through it. Note that the metal and glass substrates used in Section 6 are solid, having flat surfaces.

In order to reduce the gas temperature without increasing the gas flow and keeping a plasma length of a few millimetres we use a second waveform generator (Agilent $33220 \mathrm{~A}$ ) and apply a $20 \mathrm{kHz}$ modulation of the RF voltage with a duty cycle of $20 \%$ when using the ring electrode configuration. The plate electrode is used either in continuous mode or with the same pulse modulation as with the ring electrode.

In order to compare the three sources at least one parameter needs to be comparable for all three 
configurations. Voltage and therefore power dissipation could not be used, since the power coupling between these devices depends on the electrode configuration which changes the matching. If the same power for the continuous plate electrode is used as for the pulsed ring electrode, either the plasma is too long and too hot in the former case, or the voltage is too low to sustain a plasma in the latter case. To resolve this issue the input parameters (i.e. amplifier gain and RF voltage to the amplifier) are chosen in order to obtain the same plasma length measured between the grounded electrode and the end of the visible plasma plume, i.e. $5-6 \mathrm{~mm}$, while maintaining a gas temperature at the tip of the plasma of below $100{ }^{\circ} \mathrm{C}$. However, for all three presented cases the input power is not changed between touching and non-touching conditions.

To measure changes in the plasma induced by a substrate, different materials have been placed below the plasma jet. The distance between plasma plume and substrate has been decreased until a clear visible connection between the plasma and the substrate is observed. To maintain a stable contact, the distance between the substrate and the jet is further decreased by $2 \mathrm{~mm}$ in all cases. The gain of the amplifier and the RF voltage supplied to the amplifier have been kept the same between the "free" plasma and the plasma in contact with a substrate. A CCD-camera (Sony DSC-R1) has been used to obtain images of the free plasma comparing it with a plasma in contact with a substrate.

Figure 2 shows a simplified equivalent circuit for the plate (Fig. 2a) and the ring electrode configuration (Fig. 2b). The amplifier is represented by an alternating voltage source and a $50 \Omega$ resistor, which represents the output resistance. The matching network (a coil) is described as an inductance and a resistor. The latter includes the resistance of the wire and of the needle electrode. In the case of the plate electrode a plasma is created between the needle electrode and the substrate (if present). The plasma-electrode gap is approximated by a capacitor and a resistor in series. The substrate is represented by an impedance $\mathrm{Z}_{\text {subs }}$, which can be mainly resistive or capacitive, depending on the substrate (see Tab. 1). When no substrate is present, the plasma is capacitively coupled to the surroundings and $\mathrm{Z}_{\text {subs }}$ is a capacitor.

Since there is no plasma between the needle and the plate electrode, the effect of the plate electrode can be approximated as a capacitor in parallel with the plasma. The main difference between the plate and the ring electrode configuration is that a plasma is not only created between needle and substrate but also created between the needle and the glass tube which is surrounded by the grounded ring electrode. This system is described as a resistor and a capacitor in series, which is in parallel with the plasma connecting to the substrate.

\subsection{Power and phase shift measurements}

A voltage probe (Tektronix P5100) and a current probe (Pearson 2877) connected to an oscilloscope (Agilent Technologies DSO1024A) have been connected between

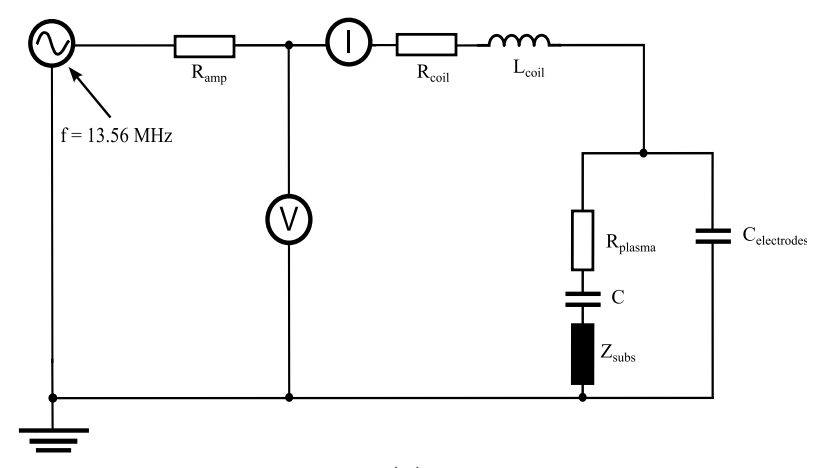

(a)

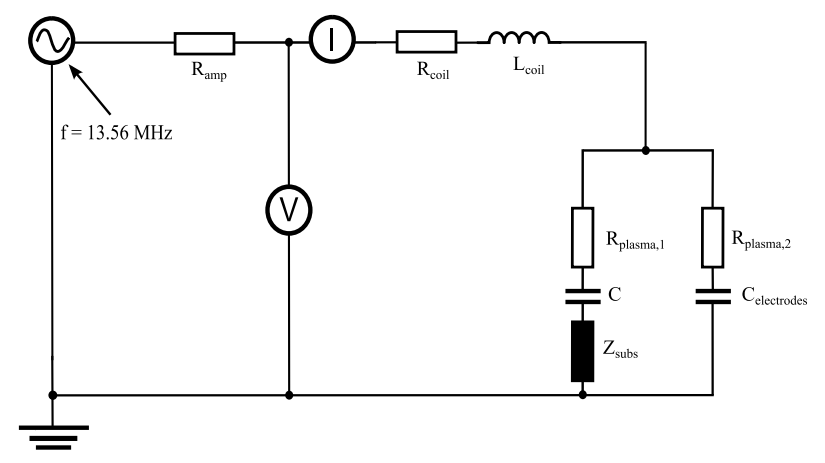

(b)

Fig. 2. Equivalent circuit of the two electrode configuration, i.e. plate (a) and ring (b) electrode.

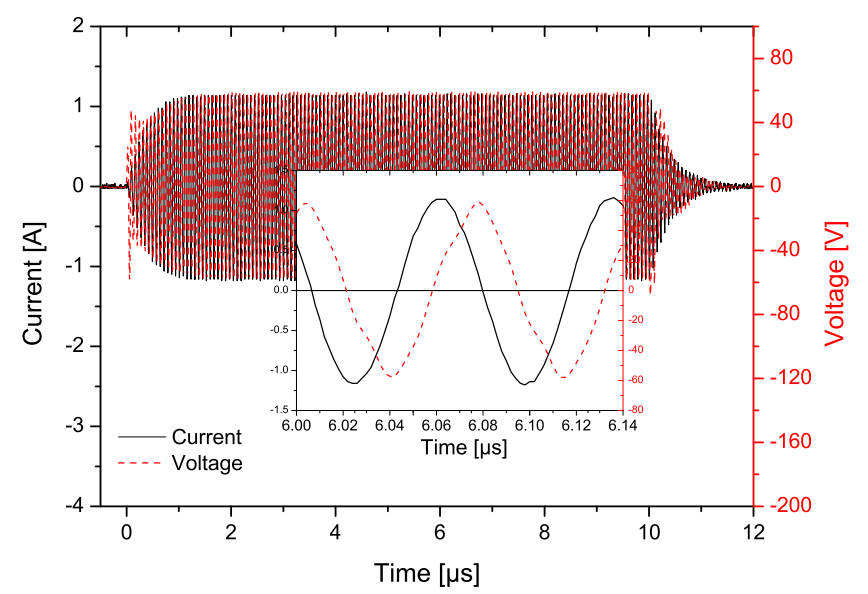

Fig. 3. Voltage and current during the pulse modulation with the ring electrode configuration without plasma. A zoomed-in view of the voltage and the current is shown as well.

the power amplifier and the matching network to measure the voltage across and the current through both the matching box and the plasma source.

Figure 3 shows an example of the voltage and current obtained for the ring electrode configuration in pulsed mode without a gas flow, i.e. without a plasma.

With $P=\frac{1}{f} \int_{t_{0}}^{t_{0}+\frac{1}{f}} U(t) I(t) d t$, the average power over one RF cycle is then calculated. In order to obtain the 


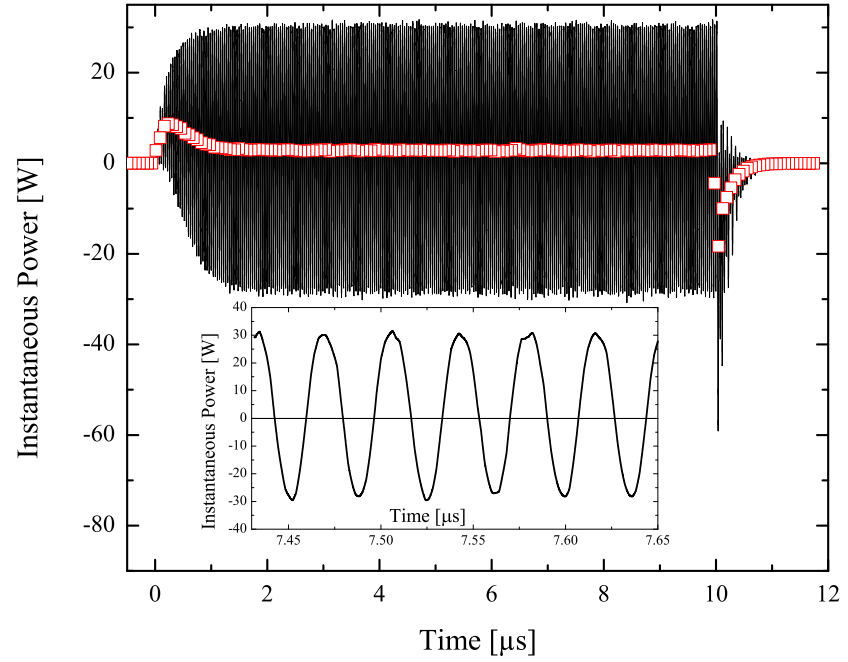

Fig. 4. Time resolved instantaneous power (black line) and the averaged power (red rectangle) without plasma, averaged over one RF cycle. A zoomed-in view of the instantaneous power is shown as well.

power dissipated only in the plasma the delay induced by the voltage and current probes and the oscilloscope have to be corrected [34], as well as the power dissipated inside the matchbox. Details can be found in reference [32].

Figure 4 shows an example of the instantaneous power, i.e. $P(t)=I(t) U(t)$ and the power dissipation averaged over one RF cycle of the circuit without an applied gas flow (no plasma) calculated from the voltage and current signal from Figure 3. Two peaks in power are visible at the beginning and the end of the pulse. These peaks correspond to the increasing and decreasing part of the voltage amplitude which indicates that energy is stored in parts of the electrical circuit and released at the end of the pulse. Calculating the energy of the peaks results in an energy of $6.76 \mu \mathrm{J}$ and $4.10 \mu \mathrm{J}$ for the first and the last peak, respectively. The constant positive power offset in between the peaks originates from the resistance of the electrical parts, most dominantly from the wire of the coil in the matching network. The average power loss in the resistive part of the setup is $(2.86 \pm 0.12) \mathrm{W}$ for the settings of Figure 4 . Similar results are obtained with the plate electrode.

Next to the power dissipation the phase shift between the voltage and current signal has also been obtained to monitor the change in impedance of the circuit. The obtained phase shift is the phase induced by the coil of the matching network, the jet geometry, the plasma and any present stray capacitances.

For the continuous plate electrode the power measurements have been validated with power measurements performed with a power meter (AR DC2600A), showing that the calculated average power corresponds to the measured forward power subtracted with the reflective power.

Note that for the time resolved ICCD images a different power amplifier is used (E \& I A-075) and the matching frequency shifted from $13.56 \mathrm{MHz}$ to $13.96 \mathrm{MHz}$.

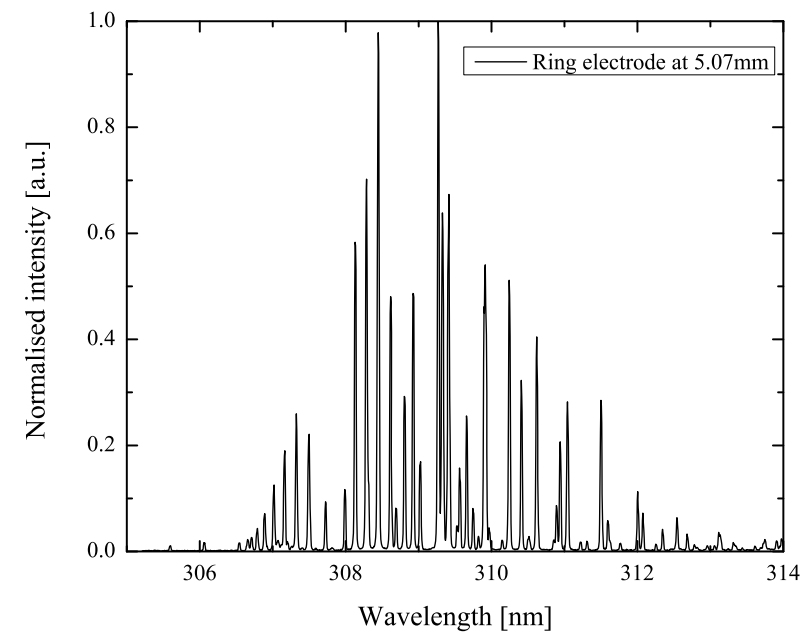

Fig. 5. Example of the spectrum from the $\mathrm{OH}$ emission band for the ring electrode configuration with no substrate approximately $5 \mathrm{~mm}$ away from the needle electrode. Plasma dissipated power $=5.2 \mathrm{~W}$.

\subsection{Time resolved ICCD images}

An ICCD camera (Stanford Computer Optics 4Picos) has been used and synchronised with the $\mathrm{kHz}$ pulse modulation to obtain time resolved images of the plasma at different delay times during the $50 \mu$ s period of the modulated RF power. The exposure time of the images was set to $100 \mathrm{~ns}$. The images are the accumulations of 1000 consecutive pulses at the same delay time. The images are synchronised with the pulse modulation but not with the $\mathrm{RF}$ frequency, meaning that each accumulation was obtained at a random phase of the $\mathrm{RF}$ cycle. This results in a measured plasma emission averaged over the RF modulation. In order to increase the spatial resolution of the image a camera lens (Tamron Japan 572D) and an additional lens with a focus of $30 \mathrm{~cm}$ have been placed between the ICCD camera and the plasma source. The images are wavelength integrated over the visible wavelength range.

\subsection{Spatial resolved gas temperature}

A 1 m-monochromator (Jobin Yvon HR 1000 M) has been used to obtain the molecular emission band of the $\mathrm{OH}(\mathrm{A}-\mathrm{X})(0,0)$ transition. The monochromator is backed by a CCD camera (SBIG ST-2000XMI-UV, pixel size $8 \mu \mathrm{m})$. Two quartz lenses $(f=30 \mathrm{~cm})$ have been aligned between the plasma jet and the monochromator to project a 1 to 1 image of the plasma jet on the entrance slit of the monochromator. An example of the emission spectrum can be seen in Figure 5 showing a normalised emission spectrum averaged axially over 30 pixels.

An example of the Boltzmann plot method corresponding to Figure 5 can be seen in Figure 6. Only the first five rotational numbers of the rotational emission band have been considered (corresponding to 6 different transitions), since it has been shown by Bruggeman et al. [35] that emission lines from higher rotational numbers do not 


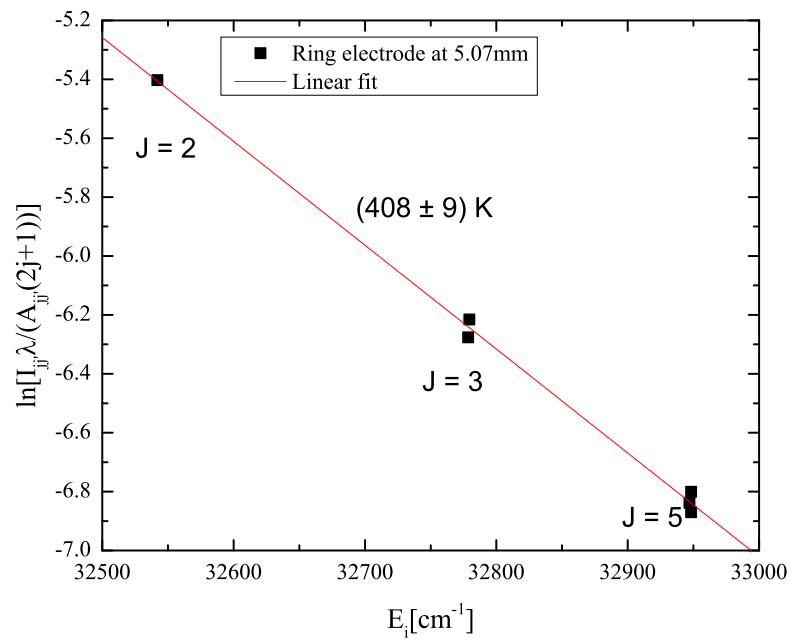

Fig. 6. Example of the Boltzmann plot from the $\mathrm{OH}$ emission band of the spectrum shown in Figure 5.

necessarily follow a thermalised Boltzmann distribution corresponding to the temperature of the gas. In previous work [32], the authors have validated this method with Rayleigh scattering. The error indicated in Figure 16 only takes into account the error of the least squares linear fit applied to the data.

The power shown in Section 4.3 and the temperature shown in Section 5 have been measured simultaneously. The CCD images have been captured at the same conditions but not during the same measurement series.

\section{Appearance of the plasma jets}

\subsection{CCD-images}

Figure 7 shows images of the plasma for the three different configurations. The first image $7 \mathrm{a}$ is the continuously driven RF plate electrode configuration. As seen here and previously $[32,36]$ the plasma is contracted showing either one stable column or one or more randomly moving filaments. As explained in $[32,37]$ losses dominated by dissociative recombination compared to losses due to diffusion are the main cause for the contraction of the plasma as the production of metastables in the dissociative recombination process leads to high densities of metastables and an efficient ionisation in the centre, with

$$
\begin{gathered}
\mathrm{Ar}_{2}^{+}+e \rightarrow \mathrm{Ar}_{m}+\mathrm{Ar} \\
\mathrm{Ar}_{m}+e \rightarrow \mathrm{Ar}^{+}+2 e
\end{gathered}
$$

The randomly created filamentation of the plasma as seen in Figure $7 \mathrm{a}$ is contributed to a relatively high absorbed power in the plasma which is then distributed into two or more randomly moving filaments rather than into one stable plasma column. However, the geometry and the high electric field at the needle tip and the presence of the dielectric glass tube do also play a role in the filamentation and the stability of the plasma. Filamentation in RF plasma jet sources have been also observed elsewhere and can exhibit self organized behaviour [38].

In Figure $7 \mathrm{~b}$ the same electrode configuration is used but the RF signal is time modulated at $20 \mathrm{kHz}$ and $20 \%$ duty cycle. In order to sustain the plasma and keep the length the same as in the continuous RF case, the power has to be increased in the case of the modulation as explained in Section 2. This leads to a higher instantaneous power in the latter case, but the average power and the gas temperature is lower than for the continuous RF driven jet (see Sects. 4.3 and 5).

The reason the plasma shows only one filament, when the voltage is time modulated, is probably due to the gas flow and the fact that the plasma is off every $50 \mu \mathrm{s}$ for $40 \mu \mathrm{s}$, leaving time for the charge in the vicinity of the needle to significantly reduce and metastable species to be blown out. This leads to a reduction in the ionisation rate at the needle tip and a preferential direction guided by the gas (and metastable) flow. This has also been observed in the case of a pulsed dc excited plasma at similar frequencies in reference [39]. The advantage of the time modulation is that the relative high electron and reactive species density (typically in RF plasmas) is present, while having a lower gas temperature due to the low duty cycle (20\%) (see also e.g. [40-42]).

The last image, Figure $7 \mathrm{c}$ shows the plasma created with the grounded ring electrode configuration. The modulation of the plasma is the same as for the grounded plate electrode configuration. With a gas flow of $1 \mathrm{slm}$ and a strong discharge between the needle and the glass tube surrounded by the grounded ring electrode, the modulation is necessary to prevent the gas from heating too much inside the glass tube, while maintaining a plasma length of a few millimetres outside of the tube. Most of the plasma is created inside the glass tube in between the needle and the grounded ring, since the electric field is the highest in this region and drops rapidly outside the tube. Due to the gas flow this plasma is then blown out into the ambient air, resulting in a more homogeneous and diffuse plasma compared to the plasmas created with the plate electrode.

In the bulk of the diffuse plasma a bright core can be seen which is created, as in the previous two cases, due to the high electric field in the axial direction caused by the sharp needle tip. Note that, if the gas flow rate is higher and becomes turbulent, the filament disappears and the length of the diffuse plasma is also shorter, similar to reference [39] and other references therein. The low gas flow of $1 \mathrm{slm}$ is chosen in order to perform treatment of liquid in small volumes with the plasma source and to keep a non-turbulent gas flow.

If a conductive substrate, such as water, is introduced to the circuit it can be seen that, if the substrate is close enough, the length of the plasma is increasing towards the substrate in all three cases and if the distance between plasma and substrate is even closer, the plasma gets in direct contact to the substrate as shown in Figure 8. For these images and the following measurements tap water with a conductivity of $250 \mu \mathrm{S} / \mathrm{cm}$ is used. The water is brought into contact with the plasma, by decreasing 


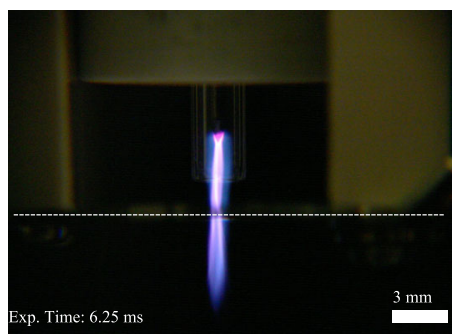

(a)

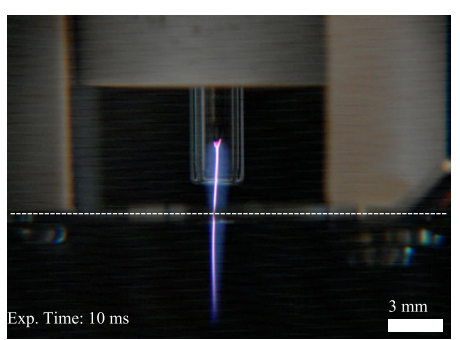

(b)

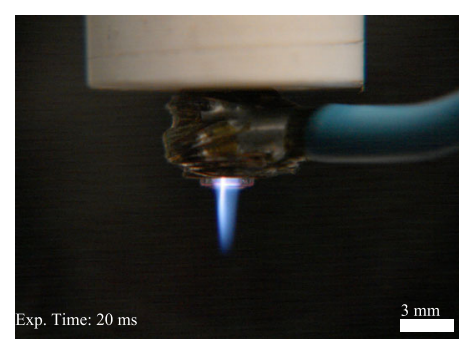

(c)

Fig. 7. CCD images of the three different plasma conditions not in contact with water (free plasma). The dashed line indicates the position of the grounded plate electrode. (a) Continuous RF driven needle with grounded plate electrode. (b) Modulated $\mathrm{RF}$ driven needle with grounded plate electrode. (c) Modulated RF driven needle with grounded ring electrode.

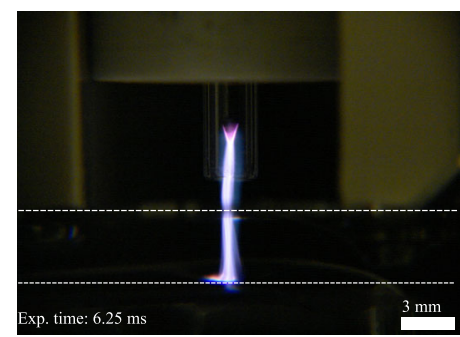

(a)

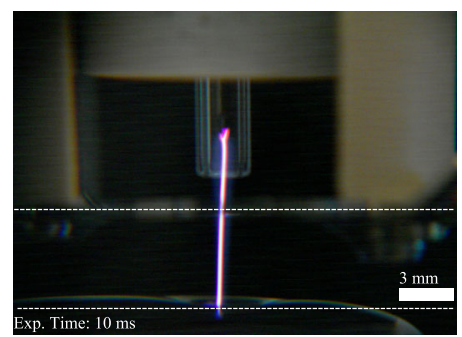

(b)



(c)

Fig. 8. CCD images of the three different plasma conditions in contact with water (touching water). The upper dashed lines indicate the position of the grounded plate electrode, if present. The lower dashed lines indicate the position of the water substrate. (a) Continuous RF driven needle with grounded plate electrode. (b) Modulated RF driven needle with grounded plate electrode. (c) Modulated RF driven needle with grounded ring electrode.

the distance between plasma and water while keeping the input parameters of the RF amplifier constant. Liquids ranging from distilled water to $\mathrm{NaCl}$-solutions with a conductivity up to $14.2 \mathrm{mS} / \mathrm{cm}$ have been used as well, however for the investigated conditions of this work no significant dependence of the conductivity of the water in power dissipation and temperature have been observed.

Figure $8 \mathrm{a}$ shows the plasma with the grounded plate electrode with continuous RF excitation in direct contact with the water substrate. Similar images have been also obtained for other plasma sources [24,38].

For the time modulated cases the plasma changes into a more intense filament (Figs. 8b and 8c). In the latter case (modulated ring electrode configuration, Fig. 8c) a strong contracted discharge column is clearly visible between the needle electrode and the water substrate, while a diffuse discharge is observed without the liquid. Both filaments in Figures $8 \mathrm{~b}$ and $8 \mathrm{c}$ are stable and are not moving randomly on the substrate as in the case for the continuously driven plasma.

Figure 9 shows an emission spectrum of the plasma, obtained coaxially, created with the ring and the plate electrode configuration at the same peak dissipated power between 200 and $1000 \mathrm{~nm}$. The setup and alignment used was the same as mentioned in reference [43]. The spectrum is normalized to the highest peak intensity and is not corrected for the wavelength dependent sensitivity of the spectrometer.

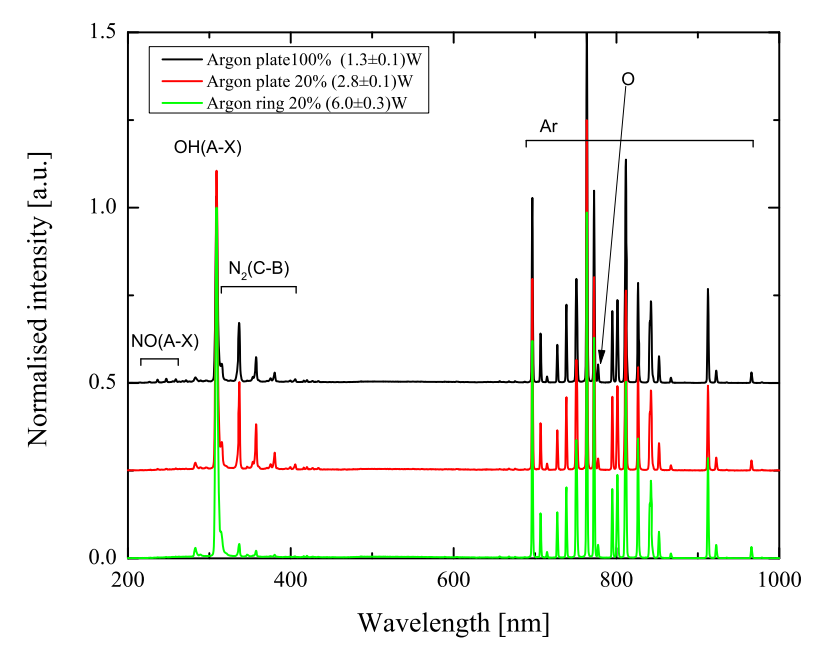

Fig. 9. Comparison of the emission spectrum of the ring electrode plasma (pulsed) and the plate electrode plasma (pulsed and continuous) for the same peak power settings as used for the temperature measurements (free plasma). An intensity offset of 0.25 and 0.5 has been added to the spectra obtained from the plate electrode configurations at $20 \%$ and $100 \%$ duty cycle, respectively.

The emission spectrum in the UV range is dominated by $\mathrm{N}_{2}(\mathrm{C}-\mathrm{B})$ in the range from 300 to $400 \mathrm{~nm}$ and $\mathrm{OH}(\mathrm{A}-\mathrm{X})(0,0)$ emission at $310 \mathrm{~nm}$. The ratio between the 


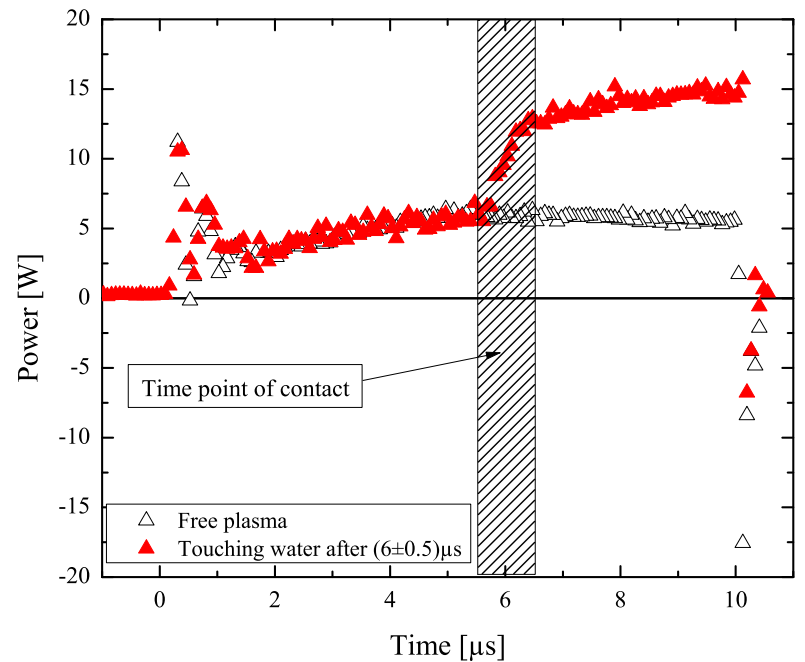

Fig. 10. Time resolved power dissipation over one period of the $\mathrm{RF}$ modulation with and without direct contact with the water substrate at $6 \mu \mathrm{s}$. The jet configuration is with a grounded plate electrode.

nitrogen emission and the $\mathrm{OH}$ peak is higher in case of the plate electrode (and highest for the continuous RF). This is to be expected since the majority of the plasma for the ring electrode configuration is created inside the glass tube (less amount of air in the plasma plume). The plate electrode plasma is sustained outside the tube, where the air concentration in the argon effluent is higher [44]. This leads to a higher emission of the nitrogen bands. Below $300 \mathrm{~nm}$ rotational bands of the $\mathrm{NO}(\gamma)$-system are visible in the case of the plate electrode configuration. In the range between 500 to $1000 \mathrm{~nm}$ atomic line emission from argon and the emission line from atomic oxygen at $777 \mathrm{~nm}$ are the most dominant peaks.

\section{Electrical and optical characteristics}

The main cause of the change of the plasma when a substrate is introduced is induced by a change of electric field (due to a change in geometry) and this results in a change in plasma impedance, altering the load of the amplifier and therefore changing the power delivered into the plasma. These effects are investigated in this section in detail by analysing the voltage, current, phase shift and power of the plasma.

\subsection{Time resolved power and ICCD images}

In Figure 10 the time resolved average power over one RF cycle is obtained for the time modulated plate electrode configuration with the plasma switched on. In this figure the power is shown for a plasma with and without direct contact with the water substrate. As explained above, the power is corrected for the power dissipation due to resistances in the electrical network. The power is not corrected

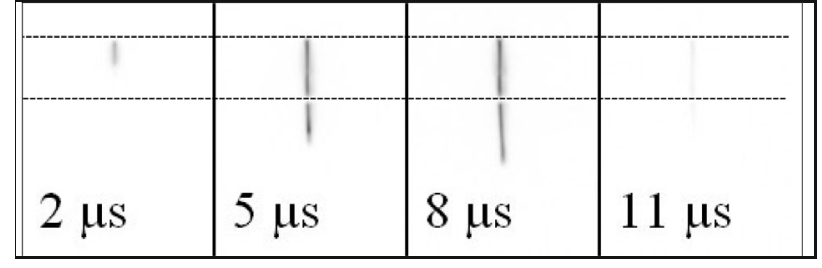

(a)

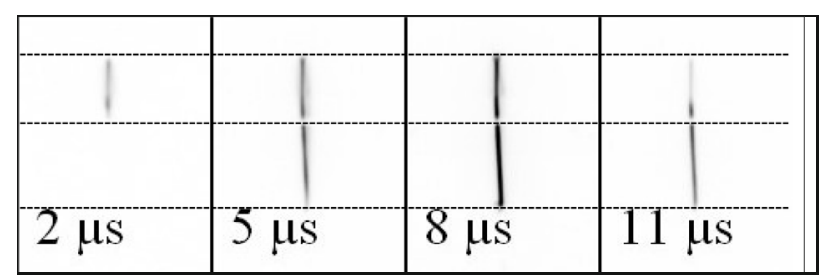

(b)

Fig. 11. Time resolved images of the time modulated grounded plate electrode configuration corresponding to the experimental conditions of Figure 10. (a) Free plasma. Exposure time $100 \mathrm{~ns}$, accumulations $=1000$, amplifier gain of ICCD $=760 \mathrm{~V}$. (b) In contact with liquid substrate. Exposure time $100 \mathrm{~ns}$, accumulations $=1000$, amplifier gain of ICCD $=760 \mathrm{~V}$.

for the reactive part (i.e. the power peaks during the on and off switching of the RF power).

For these measurements the input power and distance between plasma source and water substrate have been adjusted so that the plasma is in direct contact with the water substrate approximately half of the time. The time point when the propagating plasma reaches the water substrate is determined by taking time resolved ICCD images of the plasma (Fig. 11) and linking the time delay of the ICCD camera to the corresponding time of the currentvoltage waveforms. For these measurements the time at which the plasma reaches the water substrate is around $(6 \pm 0.5) \mu$ s after the start of the pulse as indicated in Figure 10 by the shaded area.

The time $0 \mu \mathrm{s}$ corresponds to the start of the trigger to switch on the RF voltage as shown in Figure 3. The discharge ignites at the plasma tip at a delay of $1.5 \mu \mathrm{s}$. This is in correspondence with [45]. The voltage pulses switches off after $10 \mu \mathrm{s}$ (see also Fig. 4 or Fig. 10). For the free plasma a weak emission is still visible up to $1 \mu$ s after the plasma is switched off.

Figure 11b shows the time resolved images of the same plasma setting, when in contact with the water substrate. Again, the plasma ignites around $1.5 \mu$ s after the start of the pulse and is switched off at a delay of $10 \mu \mathrm{s}$.

Looking at the dissipated power of the free plasma it can be seen that there is a linear increase in power over roughly the first $4 \mu \mathrm{s}$ starting from $2.7 \mathrm{~W}$ with a slope of $(0.82 \pm 0.03) \mathrm{W} / \mu \mathrm{s}$ before the power stays constant at $5.7 \mathrm{~W}$, for these settings. In the case of the touching plasma it is clearly visible that the moment the plasma is in contact with the substrate a second significant steep increase in power, up to a factor of 2.5 , is observed. Before the plasma reaches the water substrate the power dissipation in the plasma is the same as in the case when no 


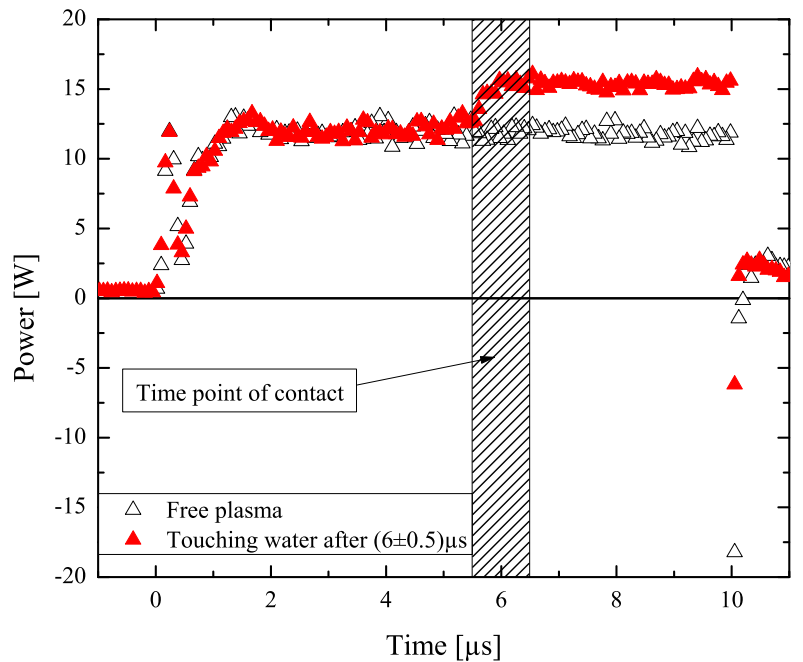

Fig. 12. Time resolved power dissipation over one pulse cycle with and without direct contact with the water substrate at $6 \mu$ s obtained with the ring electrode configuration.

substrate is in front of the plasma source. Comparing this with the corresponding ICCD images a clear increase of intensity is also visible when the plasma is connecting to the water substrate (visible in Fig. 11 at $8 \mu \mathrm{s}$ ).

From the ICCD images in Figure 11 it can be seen that the recombination lifetime is longer, when the plasma is in contact with the liquid, since the plasma emission vanishes at around $12.5 \mu \mathrm{s}$ instead after $11 \mu \mathrm{s}$ as in the case of the free plasma. The higher intensity and the longer lifetime indicate that in these cases the density of electrons and/or the metastable species increase, when the plasma is in direct contact with the liquid.

Note that a long afterglow in the emission of $\mathrm{NO}(\mathrm{A}-$ $\mathrm{X})$ is observed in reference [45], due to the fact that the $\mathrm{NO}(\mathrm{A})$ state is produced by $\mathrm{N}_{2}(\mathrm{~A})+\mathrm{NO}(\mathrm{X}) \rightarrow$ $\mathrm{NO}(\mathrm{A})+\mathrm{N}_{2}(\mathrm{X})$. The decay is a measure of the metastable lifetime of $\mathrm{N}_{2}(\mathrm{~A})$ rather than the effective radiative lifetime of NO (A). A higher density of metastables, when the plasma is in contact with the liquid could thus also contribute to the strong emission and the longer afterglow as observed in Figure 11b.

In Figure 12 the power dissipation of the ring electrode configuration is shown. In order to get to a similar length in plasma as in the case presented in Figure 10 and the time at which the plasma touches the water equal to $6 \mu \mathrm{s}$, the power is chosen to be higher than in the case of the plate electrode.

When compared with Figure 13, it can be seen that the power of the plasma increases the moment the plasma reaches the water substrate. However this increase of dissipated power does not exceed a factor of 1.3 and is thus less pronounced compared to the plate electrode geometry.

Comparing the rise time of the time resolved power between the time modulated plate electrode and the pulsed ring electrode configuration, it can be seen that the power remains constant after $2 \mu$ s for the latter case, while in the case of the plate electrode geometry, the power increases



(a)

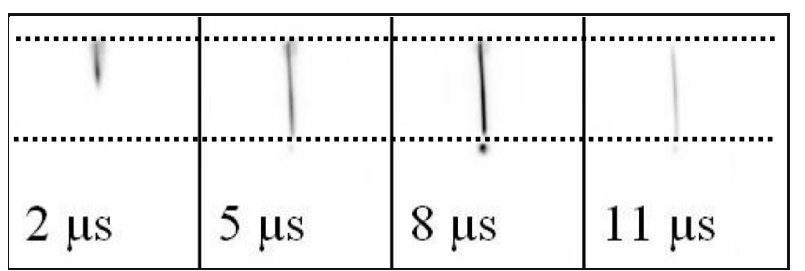

(b)

Fig. 13. Time resolved images of the pulse modulated grounded ring electrode configuration. The images correspond to the experimental conditions of Figure 12. Free plasma. (a) Exposure time $100 \mathrm{~ns}$, accumulations $=1000$, amplifier gain of ICCD $=760 \mathrm{~V}$. (b) In contact with liquid substrate. Exposure time $100 \mathrm{~ns}$, accumulations $=1000$, amplifier gain of $\mathrm{ICCD}=760 \mathrm{~V}$.

up to $6 \mu$ s before reaching a steady state value. The difference in rise time can be attributed to the difference in gap size between needle and grounded electrode, which is approximately a factor 6 larger in the case of the plate electrode configuration.

Time resolved ICCD images have been obtained for the ring electrode configuration and are shown in Figure 13. The ignition of the ring electrode plasma happens $0.5 \mu \mathrm{s}$ earlier than for the plate electrode configuration. However, the electric field is much higher in case of the ring electrode, due to the much smaller distance between the powered and the grounded electrode. Again, a large increase in intensity is visible, the moment the plasma connects to the water surface. The emission is also sustained for a longer time after the RF voltage is switched off compared to the free plasma.

\subsection{Increase of plasma length during the pulse}

From the ICCD images the propagation of the four different plasma configurations from the beginning of the pulse until the plasma is in direct contact with the liquid substrate, i.e. after a delay of $6 \mu \mathrm{s}$, has been calculated and are plotted in Figure 14.

Note that the velocity does not correspond to the velocity of the ionisation front during an RF cycle, which will be at least one order of magnitude faster [45]. Note that the gas flow is significantly slower and of the order of $10^{1} \mathrm{~m} / \mathrm{s}$. The images therefore show the slow expansion of the plasma over many RF cycles. The increase of length of the plasma plume during the time modulation can be explained by pre-ionisation linked with the number of $\mathrm{RF}$ cycles at the specific time point during a single $\mathrm{kHz}$ period [46]. 


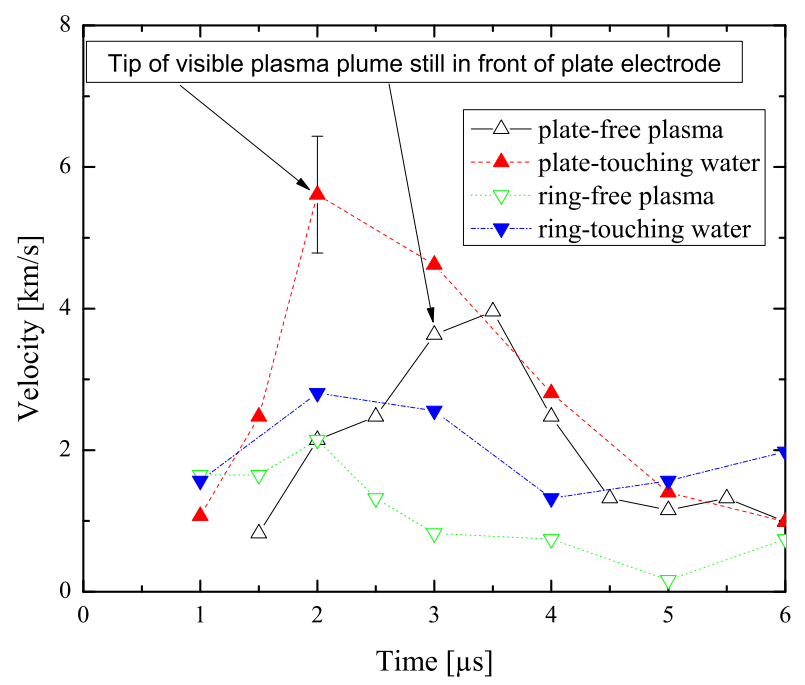

Fig. 14. Velocity of the visible plasma tip as a function of time during one modulation cycle of the different plasma electrode configurations either with or without a water substrate. The plasma reaches the water substrate at $6 \mu \mathrm{s}$. The error on all velocities, which depended on the spatial resolution of the images, is around $0.8 \mathrm{~km} / \mathrm{s}$ as indicated for one data point.

In the case of the plate electrode configuration it can be seen that the velocity in the first $3 \mu$ s increases when the liquid layer is in the vicinity of the plasma. The peak velocity is reached, both with and without the liquid, when the plasma is at the position of the plate electrode.

The velocity of the ring electrode configuration is in the first $4 \mu \mathrm{s}$ a factor 2 to 3 lower than with the plate electrode. The higher velocity of the tip of the plasma is expected due to the electric field parallel to the gas flow. The velocity is higher for the case the plasma propagates towards the liquid substrate, compared to the free plasma.

\subsection{Voltage, current and power during one RF cycle}

To further investigate the influence of the conductive substrate on electrical parameters, voltage, current and phase shift between those signals have been compared for the different conditions.

Figure 15 shows one RF cycle of the voltage and current signals of the three plasma sources either in touching or non-touching mode.

The calculated dissipated power in the plasma and phase between the voltage and current are indicated in the graphs. The error of the power consists of the systematic error due to the calculation of the phase shift originating from the uncertainty in the time delay measurement of the cables and the $U$-I-measurement. The uncertainty of the cable delay is calculated to be in the order of $0.5 \mathrm{~ns}$. The statistical error is calculated by calculating the power over around $20 \mathrm{RF}$ cycles.

Since the power depends on the phase, the closer the latter gets to zero, the smaller is the error contributed from the cable delay. The phase is measured by determining the time difference between the zero crossings of the voltage and current for $20 \mathrm{RF}$ cycles.

Figure 15a shows the voltage and current waveform for the continuous driven RF plasma with the plate electrode. The power and phase between voltage and current is indicated in the graphs. Comparing the voltage and current waveforms of the touching and non-touching mode, it can be seen that the signal is changing in voltage, current amplitude and phase-shift.

The apparent power, $P_{a p p}=U_{r m s} I_{r m s}$ is decreasing, when the plasma is in contact with the water substrate. But as the phase shift is decreasing, a more favourable matching between power amplifier and plasma source occurs, i.e. plasma + matching network impedance is closer to the impedance of the amplifier $((50+0 i) \Omega)$, and the power coupled into the plasma is higher. As expected, a similar behaviour is seen in the case of the pulsed plate electrode configuration (Fig. 15b).

Figure $15 \mathrm{c}$ shows the waveform of the ring electrode configuration. Comparing the signals of the three free plasmas, the lowest phase shift and therefore best matching is reached for the ring electrode configuration.

In this case the phase shift stays nearly constant. Due to the configuration and the strong discharge between needle and ring the load is well defined and does not change significantly with plasma conditions. The power is also increasing, which is not mainly due to the phase shift as in the two latter cases but due to the change of voltage and current amplitude, leading, contrary to the plate electrode cases, to a higher apparent power in this case and a larger power coupling to the plasma.

Comparing the ratio of the power dissipation in the touching and non-touching mode of the three investigated conditions the plate electrode is showing the biggest change, while the ring electrode shows the smallest relative change as also seen in the previous section (Tab. 2).

When the plasma is in contact with a conductive substrate, we assume that the main change is a drop in plasma resistance, possibly due to a higher electron density. This leads to a favourable matching leading to more power coupled into the load, resulting in a higher dissipated power and a reduction in phase-shift. This effect is less dominant for the ring electrode since the majority of the active plasma is created in between the electrodes, and is less affected by the surroundings.

\section{Spatial resolved gas temperature}

A change in power dissipation leads to a variety of changes of several plasma parameters. As the heat flux to a treatment substrate is a very important parameter for biological applications, the spatial resolved gas temperature of the three different cases presented in Figure 15 is shown in Figure 16.

The gas temperature is obtained by the rotational temperature of the $\mathrm{OH}(\mathrm{A}-\mathrm{X})(0,0)$ rotational emission band. Figure 16 shows either the gas temperature without any substrate or the gas temperature when the plasma is in 
This is the Accepted Manuscript version -post-print- of an article accepted for publication in The European Physical Journal D. This article may be downloaded for personal use only. Any other use requires prior permission of the authors and Springer Nature. This article may be found at doi:10.1140/epjd/e2014-40430-3.

Springer Nature is not responsible for any errors or omissions in this version of the manuscript or any version derived from it.

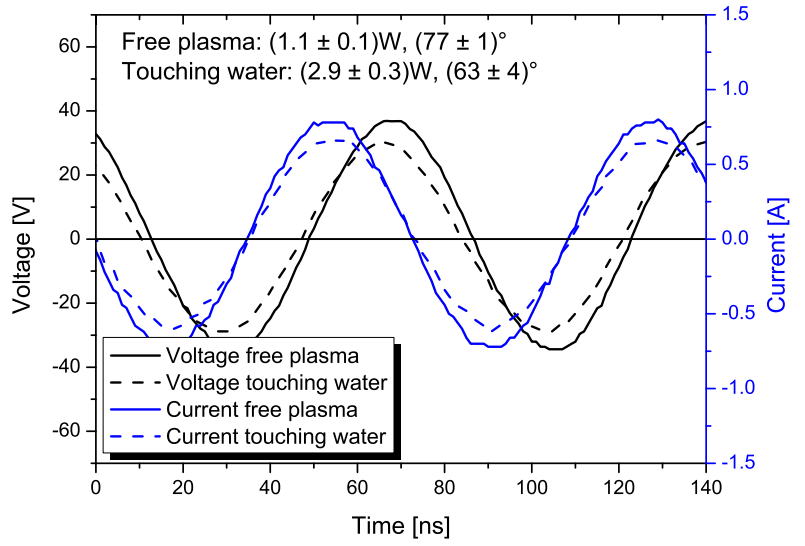

(a)

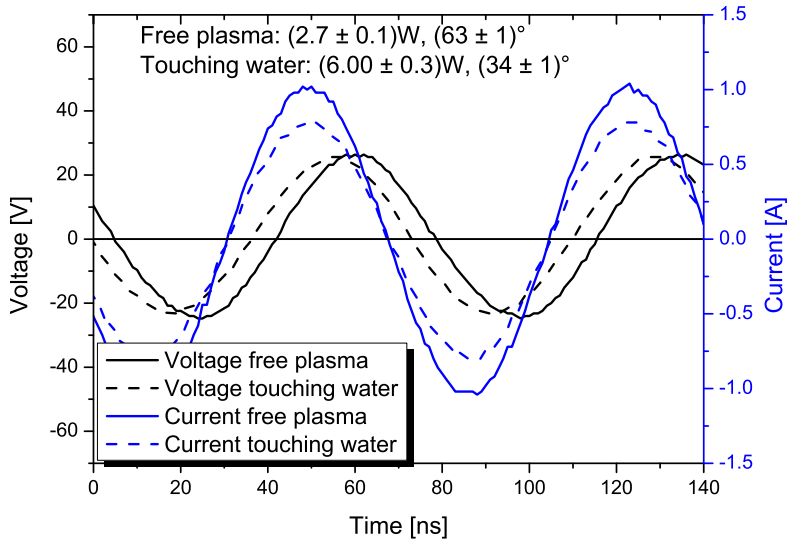

(b)

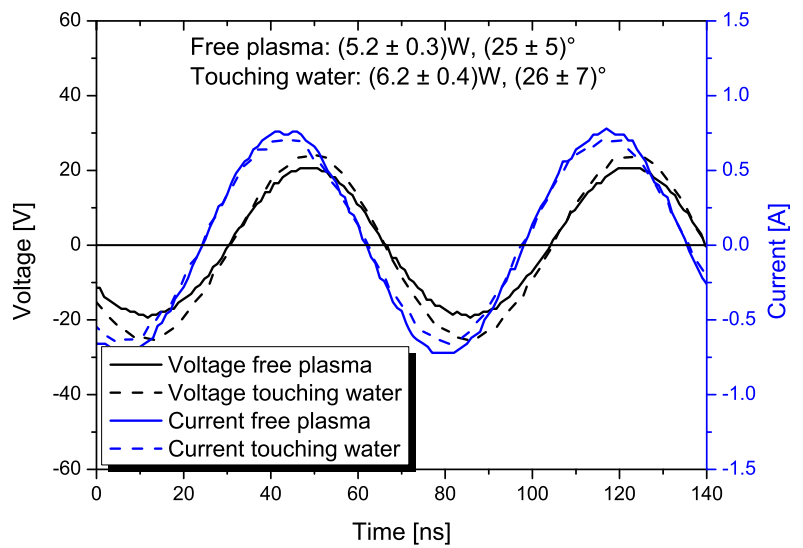

(c)

Fig. 15. Voltage and current waveforms of the three different investigated settings for touching and non-touching mode. (a) Continuous RF driven needle with grounded plate electrode. (b) Modulated RF driven needle with grounded plate electrode. (c) Modulated RF driven needle with grounded ring electrode.

Table 2. Plasma dissipated power, apparent power and phase shift of the six different conditions investigated.

\begin{tabular}{cccc}
\hline Electrode & Plasma power $[\mathrm{W}]$ & Apparent power [W] & Phase $\left.{ }^{\circ}\right]$ \\
\hline Plate (cont.) free & $1.1 \pm 0.2$ & $14.4 \pm 0.5$ & $77 \pm 1$ \\
Plate (cont.) touching & $2.9 \pm 0.2$ & $10.1 \pm 0.4$ & $63 \pm 4$ \\
\hline Plate (mod.) free & $2.7 \pm 0.2$ & $13.5 \pm 0.5$ & $63 \pm 1$ \\
Plate (mod.) touching & $6.0 \pm 0.2$ & $10.0 \pm 0.4$ & $34 \pm 1$ \\
\hline Ring (mod.) free & $5.2 \pm 0.1$ & $7.6 \pm 0.4$ & $25 \pm 5$ \\
Ring (mod.) touching & $6.2 \pm 0.1$ & $8.5 \pm 0.4$ & $26 \pm 7$ \\
\hline
\end{tabular}

contact with water substrate at a distance of approximately $7-9 \mathrm{~mm}$, depending on the configuration. The same input parameters for the RF amplifier have been used as in the previous section.

Figure 16a shows the spatial resolved rotational temperature for the continuous RF driven plate electrode configuration starting from the needle electrode on the left side of the graph until the position the plasma is touching the water substrate on the right side of the graph.

When the plasma touches the water, the gas temperature is consistently higher, compared to the gas temperature of the free plasma. In the latter case the temperature inside the glass tube is around $380 \mathrm{~K}$ and rises after it leaves the glass tube to around $440 \mathrm{~K}$ before it reaches a steady state at $380 \mathrm{~K}$. The increase of temperature once the plasma leaves the glass electrode can be explained by the admixture of air, i.e. by adding molecules, more chemical reactions which transform excess energy from the plasma to the heating of the gas are added into the system increasing the thermal output. A less pronounced effect of this has been also observed by Zhang et al. [47]. Note that an overestimate of the rotational temperature due to argon ions and metastables is unlikely. If ions and metastables would influence the rotational temperature of $\mathrm{OH}(\mathrm{A})$ in this case, this effect would be more pronounced in the far afterglow of the plasma due to increased 


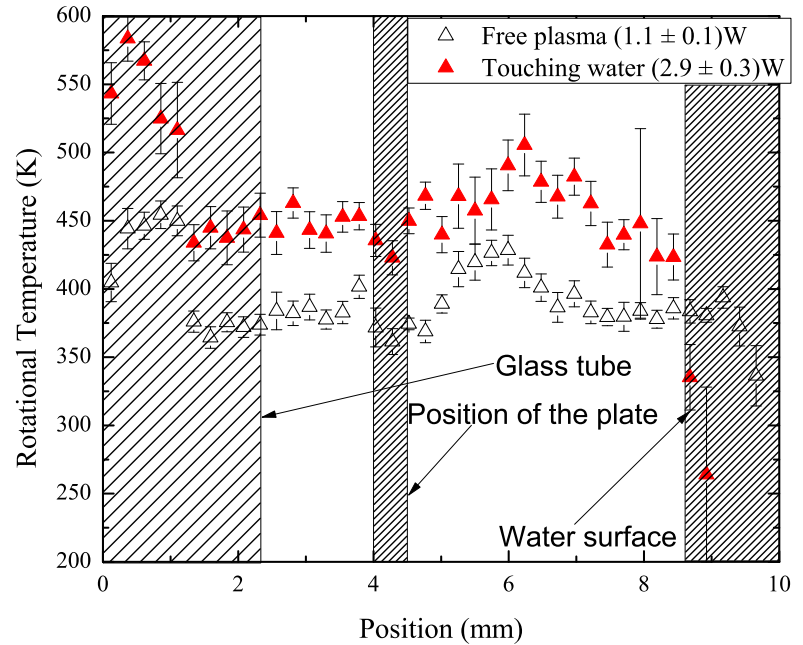

(a)

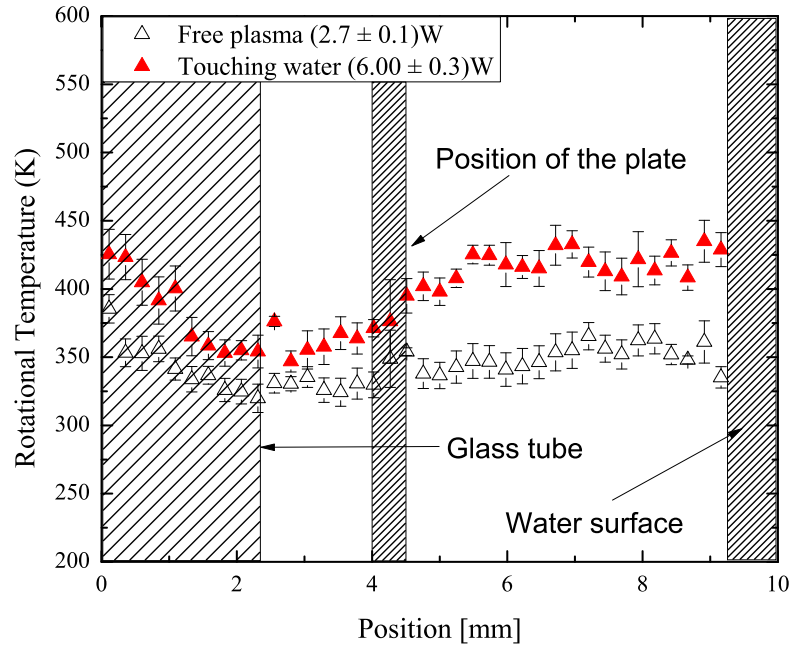

(b)



(c)

Fig. 16. Spatial resolved gas temperature in axial direction as obtained from the rotational temperature of the $\mathrm{OH}(\mathrm{A}-\mathrm{X})(0,0)$ emission band. The experimental conditions are the same as in figure 15. (a) Continuous RF driven needle with grounded plate electrode. (b) Modulated RF driven needle with grounded plate electrode. (c) Modulated RF driven needle with grounded ring electrode.

collisional quenching by air and a reduced effective lifetime of the excited state, as shown for a helium plasma by Chan et al. [48]. However, this is not the case in the used configuration, as the rotational temperature drops at the tip of the plasma. Furthermore, as mentioned above, only the lowest rotational numbers have been used for the Boltzmann-plot as has been shown by Motret et al. [49] and Bruggeman et al. [50], that at least transitions of the low rotational numbers are thermalised and corresponding to the gas temperature.

For the touching plasma, the temperature is around $50 \mathrm{~K}$ higher than in the case of the free plasma. This is to be expected since the plasma power is also higher.

The advantage of the time modulated plasma (shown in Fig. 16b) becomes clear from the gas temperature in the case of the undisturbed plasma. Although the instantaneous power is higher, the average power is reduced due to the modulation, leading to a lower gas temperature compared to the continuously driven grounded plate electrode configuration.

However, the advantage of modulation is changed the moment the plasma is in direct contact with the water substrate. The gas temperature increases by approximately $80 \mathrm{~K}$ in this case, from 350 to $430 \mathrm{~K}$. The increase of the gas temperature and power is similar as in the former case, although the gas temperature difference is getting larger closer to the water electrode. This is consistent with the fact that the plasma is most influenced by the water substrate between the plate electrode and the water.

Figure 16c shows the spatial resolved gas temperature for the grounded ring electrode configuration. For both conditions a maximum of the gas temperature is found 


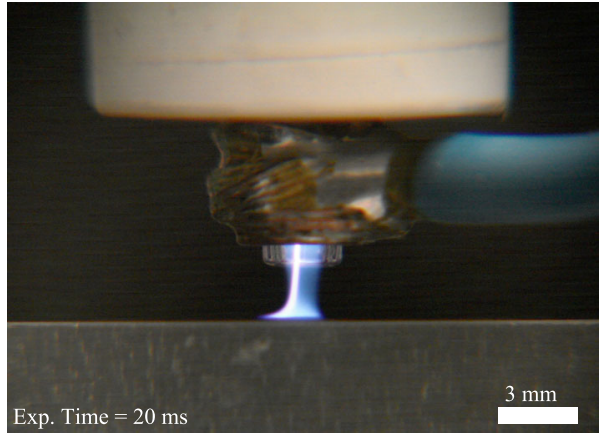

(a)

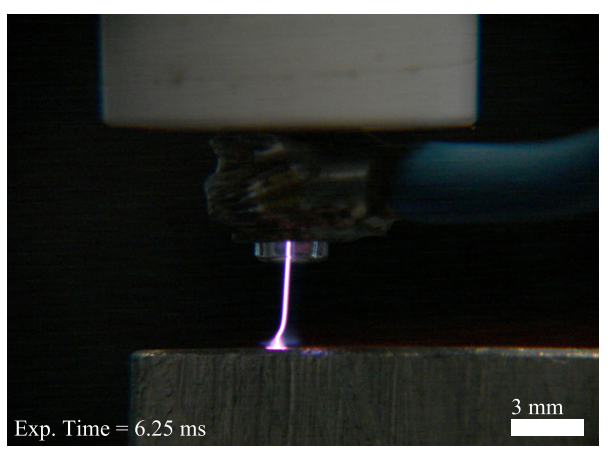

(b)

Fig. 17. CCD images of the plasma with the ring electrode configuration in contact with a glass (a) and a metal substrate (b). (a) Modulated RF driven needle with grounded ring electrode in contact with glass. Exposure time $=20 \mathrm{~ms}$. (b) Modulated RF driven needle with grounded ring electrode in contact with metal. Exposure time $=6.25 \mathrm{~ms}$.

between 4 and $5 \mathrm{~mm}$ behind the glass tube and the ring electrode in the order of $440 \mathrm{~K}$ and $460 \mathrm{~K}$ for the undisturbed and the touching plasma, respectively. Afterwards, the gas temperature drops down to $340 \mathrm{~K}$ at the end of the plasma plume. The power and average gas temperature are higher than the modulated plate electrode configuration, indicating again that the discharge is the strongest inside the glass tube and only a small fraction is created behind the grounded ring electrode. A higher temperature when the discharge is in contact with the water substrate is also observed in this configuration, although the increase is smaller the temperature drops after $5 \mathrm{~mm}$ to the same level as with the free plasma.

\section{Different materials in contact with the ring electrode configuration}

In this section further examples for the ring electrode configuration are shown in contact with a dielectric material (glass) and metal.

Images of the two conditions are shown in Figure 17. These images illustrate, that the plasma does not change significantly when in contact with a dielectric. In contact with metal, the diffuse part of the discharge disappears

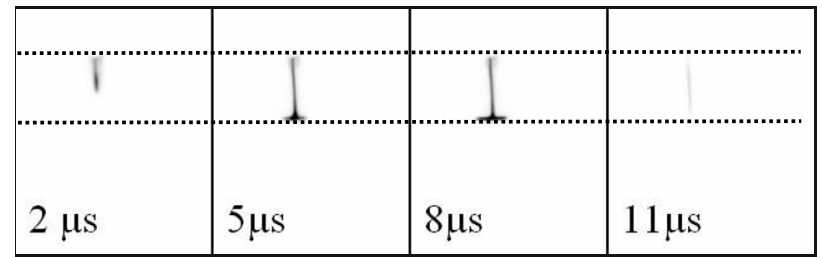

(a)

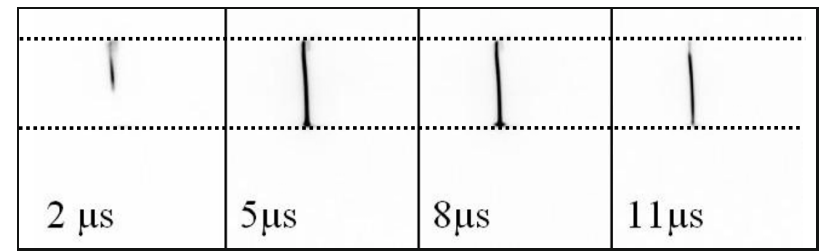

(b)

Fig. 18. ICCD images of the plasma with the ring electrode configuration in contact with a glass (a) and a metal substrate (b). The dotted lines indicate the end of the ring electrode and the position of the glass and metal substrate, respectively. The power is shown in Figure 19. (a) Modulated $\mathrm{RF}$ driven needle with grounded ring electrode in contact with glass. (b) Modulated RF driven needle with grounded ring electrode in contact with metal.

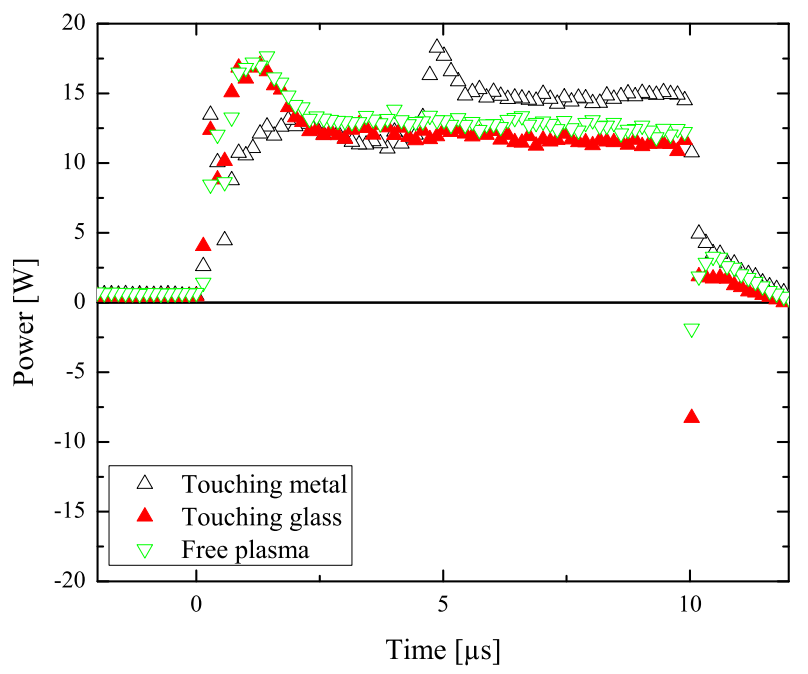

Fig. 19. Time resolved power, averaged over one RF cycle for a free plasma, a plasma touching a glass and metal substrate. The experimental conditions are the same as in Figure 18.

completely and a plasma filament, similar to the modulated plate electrode geometry, is observed. This indicates that, if the distance between a conductive substrate and the needle electrode is close enough, the ring electrode does not play a significant role as electrode any more.

Figures 18 and 19 show time resolved ICCD images and the corresponding time resolved power, for the plasma in contact with glass, metal and the free plasma. The images of the free plasma are shown in Figure 13a. As expected, the plasma touching the glass plate (which has a much lower electrical conductivity as tap water and aluminium) does not show any significant difference in power, 


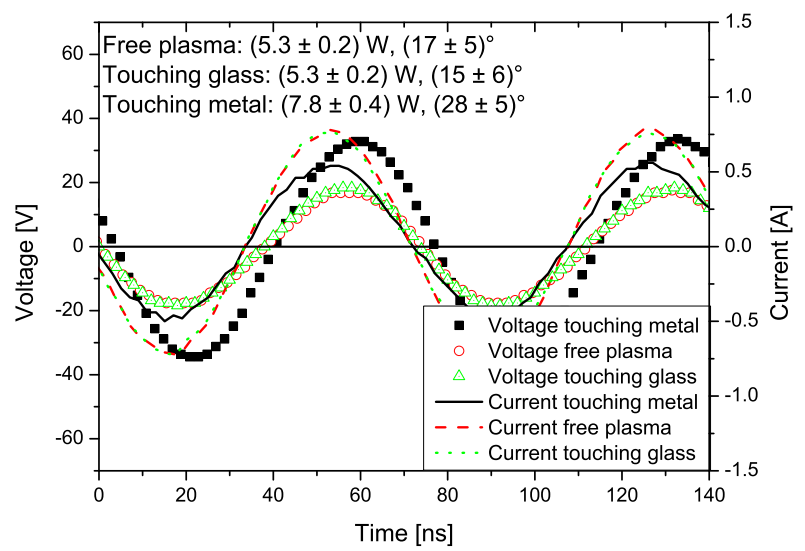

Fig. 20. Voltage and current waveform for a free plasma and a plasma touching a glass or metal substrate. The plasma dissipated power and phase shift are also given.

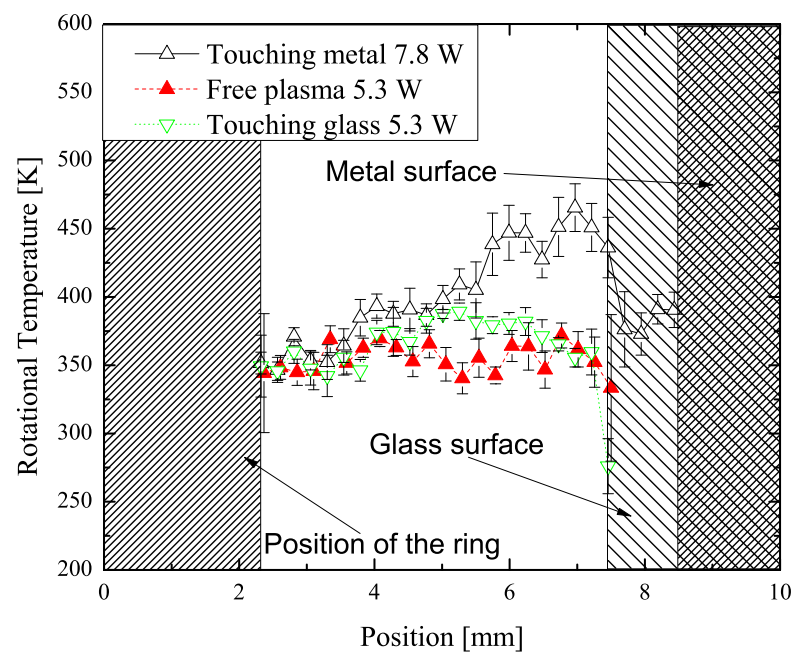

Fig. 21. Spatial resolved gas temperature for a free plasma, a plasma touching a glass or metal substrate. The experimental conditions are the same as in Figure 20.

compared to the free plasma. The plasma touching the metal substrate shows similar behaviour as in the plasma in contact with the water substrate, i.e. a higher power and higher emission, when in contact with the water (at $5 \mu \mathrm{s}$ ).

The electrical characteristics during one RF pulse for the three conditions are shown in Figure 20. When the plasma is in direct contact with the metal substrate a significant decrease in current is seen. It is believed that in this case the main current is going through the substrate and not the ring electrode. This is motivated by Figure $18 \mathrm{~b}$ which shows a single filament and no diffuse plume superimposed in the effluent which is observed in the other cases and is due to the presence of a strong axial discharge between needle and ring electrode.

Figure 21 shows the spatial resolved gas temperature, corresponding to the electrical characteristics. As can be expected from the images, no significant change in electrical characteristics or in the gas temperature are observed if the plasma is in contact with a glass substrate, compared to the free plasma. If metal is used as substrate, power and gas temperature are increasing (with a maximum gas temperature approximately $1 \mathrm{~mm}$ before the metal substrate) as seen with the water substrate. The phase shift is slightly higher when the plasma is in contact with the metal substrate, compared to the other two cases, as well as the apparent power.

\section{Conclusion}

In this contribution measurements of the plasma dissipated power (time resolved) and the gas temperature (spatial resolved) of an atmospheric pressure $\mathrm{RF}$ driven plasma jet operated with three different configurations while in contact with various substrates of different conductivity and permittivity (water, glass, metal) are presented. It is shown that, if a conductive substrate is too close to the plasma plume, the power dissipation in the plasma and the gas temperature increases.

Time resolved measurements of the plasma morphology and the power indicated that the increase of power is only starting the moment the plasma touches the conductive substrate. An increase up to a factor of 3 in power has been observed for the plate electrode configuration, while a factor less then 1.5 is observed for the ring electrode configuration. The change in impedance of the plasma source leading to a more favourable matching is shown to be the main reason of the observed change in electrical characteristics. In addition when in direct contact with a conductive substrate, the gas temperature of the plasma jet increases in all observed cases with a maximum of up to $80 \mathrm{~K}$.

The electrical properties of the material which is treated in direct contact with the plasma strongly influences the plasma (parameters) and diagnostics need to be applied for identical conditions.

This also indicates, if conductive meshes are used, as for example to reduce the charge flux onto the substrate in biological experiments, it is likely that the plasma is changing (e.g. increase of the density of charged and reactive species) resulting in an increased flux of reactive species.

The authors would like to thank Eddie van Veldhuizen and Ronny Brandenburg for fruitful discussions. P.J.B. acknowledges funding from the Dutch Technology Foundation (STW) and S.H. acknowledges funding from the Foundation for Fundamental Research on Matter (FOM).

\section{References}

1. L. Bárdos, H. Baránková, Thin Solid Films 518, 6705 (2010)

2. J. Heinlin, G. Morfill, M. Landthaler, W. Stolz, G. Isbary, J.L. Zimmermann, T. Shimizu, S. Karrer, J. Dtsch. Dermatol. Ges. 8, 968 (2010)

3. M. Laroussi, IEEE Trans. Plasma Sci. 37, 714 (2009) 
4. S. Kalghatgi et al., in Plasma Assisted Decontamination of Biological and Chemical Agents, edited by A. Fridman, S. Güçeri (Springer Science-Business Media B.V., 2008), pp. $173-181$

5. G.B. McCombs, M.L. Darby, Int. J. Dent. Hygiene 8, 10 (2010)

6. G. Lloyd, G. Friedman, S. Jafri, G. Schultz, A. Fridman, K. Harding, Plasma Process. Polym. 7, 194 (2010)

7. H.W. Lee, G.Y. Park, Y.S. Seo, Y.H. Im, S.B. Shim, H.J. Lee, J. Phys. D 44, 053001 (2011)

8. M.G. Kong, G. Kroesen, G. Morfill, T. Nosenko, T. Shimizu, J. Van Dijk, J.L. Zimmermann, New J. Phys. 11, 115012 (2009)

9. G. Morfill, M. Kong, J. Zimmermann, New J. Phys. 11, $115011(2009)$

10. M. Laroussi, IEEE Trans. Plasma Sci. 24, 1188 (1996)

11. K. Kelly-Wintenberg, T.C. Montie, C. Brickman, J.R. Roth, A.K. Carr, K. Sorge, L.C. Wadsworth, P.P. Tsai, J. Ind. Microbiol. Biotechnol. 20, 69 (1998)

12. A. Schütze, J.Y. Jeong, S.E. Babayan, J. Park, G.S. Selwyn, R.F. Hicks, IEEE Trans. Plasma Sci. 26, 1685 (1998)

13. X. Lu, M. Laroussi, V. Puech, Plasma Sources Sci. Technol. 21, 34005 (2012)

14. G.Y. Park, S.J. Park, M.Y. Choi, I.G. Koo, J.H. Byun, J.W. Hong, J.Y. Sim, G.J. Collins, J.K. Lee, Plasma Sources Sci. Technol. 21, 043001 (2012)

15. H.E. Wagner, R. Brandenburg, K.V. Kozlov, A. Sonnenfeld, P. Michel, J.F. Behnke, Vacuum 71, 417 (2003)

16. G.E. Morfill, T. Shimizu, B. Steffes, H.U. Schmidt, New J. Phys. 11, 115019 (2009)

17. M. Kuchenbecker, N. Bibinov, A. Kaemlimg, D. Wandke, P. Awakowicz, W. Viöl, J. Phys. D 42, 045212 (2009)

18. D. Dobrynin, G. Fridman, G. Friedman, A. Fridman, New J. Phys. 11, 115020 (2009)

19. N.Y. Babaeva, M.J. Kushner, J. Phys. D 43, 185206 (2010)

20. G. Fridman, A.D. Brooks, M. Balasubramanian, A. Fridman, A. Gutsol, V.N. Vasilets, H. Ayan, G. Friedman, Plasma Process. Polym. 4, 370 (2007)

21. E.P. van der Laan, E. Stoffels, M. Steinbuch, Plasma Sources Sci. Technol. 15, 582 (2006)

22. Y. Sakiyama, D.B. Graves, E. Stoffels, J. Phys. D 41, 95204 (2008)

23. Y. Kim, M.S. Cha, W.H. Shin, Y.H. Song, J. Korean Phys. Soc. 43, 732 (2003)

24. S. Bornholdt, M. Wolter, H. Kersten, Eur. Phys. J. D 60 , $653(2010)$

25. E. Stoffels, I.E. Kieft, R.E.J. Sladek, L.J.M. van den Bedem, E.P. van der Laan, M. Steinbuch, Plasma Sources Sci. Technol. 15, S169 (2006)

26. M. Dünnbier, A. Schmidt-Bleker, J. Winter, M. Wolfram, R. Hippler, K.D. Weltmann, S. Reuter, J. Phys. D 46, $435203(2013)$

27. D. Maletić, N. Puač, S. Lazović, G. Malović, T. Gans, V. Schulz-von der Gathen, Z.L. Petrović, Plasma Phys. Control. Fusion 54, 124046 (2012)
28. U. Kaatze, J. Chem. Eng. Data 34, 371 (1989)

29. Conductivity and Resistivity Values for Aluminum \& Alloys, http://www.ndt-ed.org/

30. http://www.ohara-inc.co.jp/en/product/ electronics/gd-fht.html

31. http://www.ultiquestcom.com/products/optics/ optical-glass-nbk7-b270-and-others.html

32. S. Hofmann, A.F.H. van Gessel, T. Verreycken, P. Bruggeman, Plasma Sources Sci. Technol. 20, 065010 (2011)

33. J. Walsh, M. Kong, Appl. Phys. Lett. 93, 111501 (2008)

34. V. Léveillé, S. Coulombe, Meas. Sci. Technol. 17, 3027 (2006)

35. P. Bruggeman, F. Iza, P. Guns, D. Lauwers, M.G. Kong, Y.A. Aranda-Gonzalvo, C. Leys, D.C. Schram, Plasma Sources Sci. Technol. 19, 15016 (2010)

36. S. Hofmann, P. Bruggeman, IEEE Trans. Plasma Sci. 39, 2 (2011)

37. E. Castaños Martínez, M. Moisan, Y. Kabouzi, J. Phys. D 42, 012003 (2009)

38. J. Schäfer, R. Foest, A. Ohl, K.D. Weltmann, Plasma Phys. Control. Fusion 51, 124045 (2009)

39. S. Hofmann, A. Sobota, P. Bruggeman, IEEE Trans. Plasma Sci. 40, 2888 (2012)

40. D.B. Kim, S.Y. Moon, H. Jung, B. Gweon, W. Choe, Phys. Plasmas 17, 053508 (2010)

41. N. Balcon, A. Aanesland, R. Boswell, Plasma Sources Sci. Technol. 16, 217 (2007)

42. R. Ye, T. Ishigaki, T. Sakuta, Plasma Sources Sci. Technol. 14, 387 (2005)

43. C.A.J. van Gils, S. Hofmann, B.K.H.L. Boekema, R. Brandenburg, P.J. Bruggeman, J. Phys. D 46, 175203 (2013)

44. A.F.H. van Gessel, Ph.D. thesis, Eindhoven University of Technology, 2013

45. A.F.H. van Gessel, K.M.J. Alards, P.J. Bruggeman, J. Phys. D 46, 265202 (2013)

46. A. Sobota, J. Van Dijk, M. Haverlag, J. Phys. D 44, 224003 (2011)

47. S. Zhang, W. van Gaens, A.F.H. van Gessel, S. Hofmann, E. van Veldhuizen, A. Bogaerts, P. Bruggeman, J. Phys. D 46, 205202 (2013)

48. G.C.Y. Chan, J.T. Shelley, J.S. Wiley, C. Engelhard, A.U. Jackson, R.G. Cooks, G.M. Hieftje, Anal. Chem. 83, 3675 (2011)

49. O. Motret, C. Hibert, S. Pellerin, J.M. Pouvesle, J. Phys. D 33, 1493 (2000)

50. P. Bruggeman, D.C. Schram, M.G. Kong, C. Leys, Plasma Process. Polym. 6, 751 (2009) 